\title{
Experimental Evidence of Charge Transfer in a Functionalized Hexa- vanadate. A High Resolution X-ray Diffraction Study
}

\author{
Xiao Xu ${ }^{\mathrm{a}, \mathrm{b}}$, Anne Spasojević-de Biréa,b*, Nour Eddine Ghermania,b,c, Yongge Weid, Sladjana Nova- \\ kovića,e, Nada Bošnjaković-Pavlovića,f, Pingfan Wug \\ a CentraleSupelec, Campus de Chatenay, Grande Voie des Vignes, 92295 Châtenay-Malabry, France \\ b Laboratory "Structures Propriétés et Modélisation des Solides" (SPMS), UMR CNRS 8580, Grande Voie des Vignes, \\ 92295 Châtenay-Malabry, France \\ c Institut Galien Paris Sud, UMR CNRS 8612, Université Paris-Sud, Faculté de Pharmacie, 5, rue Jean-Baptiste Clé- \\ ment, 92296 Châtenay-Malabry, France \\ d Department of Chemistry, Tsinghua University, 100084 Beijing, China \\ e Laboratory for Theoretical and Condensed Matter Physics, Institute Vinča, 11000 Belgrade, Serbia \\ f Faculty of Physical Chemistry, University of Belgrade, Studentski trg 12-16, 11158 Belgrade, Serbia \\ g Institute of POM-based Materials, Hubei University of Technology, The Synergistic Innovation Center of Catalysis \\ Materials of Hubei Province, 430086 Wuhan, Hubei Province, China
}

\begin{abstract}
A high resolution X-ray diffraction study has been carried out on $\left[\left(\mathrm{C}_{4} \mathrm{H}_{9}\right)_{4} \mathrm{~N}\right]_{2}$ $\left[\mathrm{V}_{6} \mathrm{O}_{13}\left\{\left(\mathrm{OCH}_{2}\right)_{3} \mathrm{CCH}_{2} \mathrm{OCCH}_{2} \mathrm{CH}_{3}\right\}_{2}\right](\mathrm{V} 6-\mathrm{C} 3)$ at $100 \mathrm{~K}$. V6 core concentrates negative charge, leading to a strong polarization of the anion. Nucleophilic region localized near the organic moiety and electrophilic region in the vicinity of the V6 core provide an overall description of charge-transfer behavior.
\end{abstract}

Charge transfer materials have attracted increasing attentions in recent years due to their roles in the fields of semiconductors, ${ }^{1}$ solar cells, 2 metal organic frameworks (MOFs), ${ }^{3}$ fluorescent materials, ${ }^{4}$ and catalysts. 5 According to Mulliken's theory, ${ }^{6}$ charge transfer (CT) system is formed between an electron donor (D) and an electron acceptor (A), D and A could be two different molecules or ions, leading to intermolecular CT, or separate moieties within a large molecule, leading to intramolecular charge transfer (ICT). 4 Polyoxometalates (POMs), a large group of early transition metal oxide clusters, are extensively studied due to their diversified structures and wide-ranging properties. 7 POMs are well-known electron acceptors in CT materials prepared with organic donors, which is based on the ability of POMs to act as electron reservoirs. ${ }^{8}$ For example, the CT materials containing the spherical POM acceptors (Lindqvist-type $\left[\mathrm{Mo}_{6} \mathrm{O}_{19}\right]^{2-}$, Keggintype $\left[\mathrm{SiMo}_{12} \mathrm{O}_{40}\right]^{4-}$ ) and planar arene donors exhibit unique structures, potential nonlinear optical and ferromagnetic properties. 9 TTF-POMs CT materials (TTF means tetrathiafulvalene) show semiconductor properties and have potential application in semiconductor devices. 8b, 10 Functionalized hexavanadates (V6) in which the inorganic V6 core is covalently bonded with organic ligands, form one of the most interesting subgroups. Functionalized hexavanadates (V6) present nanoscale superoctahedral cluster-core structures, fascinating electronic and magnetic properties, various thermodynamically stable redox isomers, and potential catalytic capabilities. ${ }^{11}$ Recently, our co-workers firstly found that the functionalized V6 clusters hybridized with hydrophobic groups show unexpected fluorescent properties probably due to their ligand-to-metal charge transfer (LMCT) which might inspire a new mechanism for fluorescence emission. ${ }^{12} \mathrm{How}$ ever, understanding such a CT behavior and fluorescence mechanism of these functionalized hexavanadates is still a formidable challenge. Experimental charge density, determined from high resolution single crystal X-ray diffraction, has become a useful tool to investigate the charge density distribution, topological properties, AIM (Atom in Molecule) charges, electrostatic potential, source function, etc. within the crystalline states. ${ }^{13}$ Enlighten by the reported works of POVbased fluorescent materials involving oxide-to-vanadium charge transfer and intervalence charge transfer transitions, 14 the investigations on CT behavior between POM acceptor and organic donor could provide a new insight into fluorescence mechanism of the functionalized hexavanadates. It has been established that accurate charge density and electrostatic properties can give precise information on the CT behavior especially in the following aspects: i) the regions where the electrostatic potential is negative / positive give an idea of the A and D groups, respectively, ii) the AIM charges of the different groups determine the amount of the charge transferred between D and A. ${ }^{15}$ In the present study, we report the atomic net charge and electrostatic properties of a functionalized hexavanadate, $\left[\left(\mathrm{C}_{4} \mathrm{H}_{9}\right)_{4} \mathrm{~N}\right]_{2}\left[\mathrm{~V}_{6} \mathrm{O}_{13}\left\{\left(\mathrm{OCH}_{2}\right)_{3} \mathrm{CCH}_{2} \mathrm{OCCH}_{2} \mathrm{CH}_{3}\right\}_{2}\right]$, in further text given as V6-C3 (Fig 1). This work represents the first experimental charge density study on an POV-based organic-inorganic hybrid compound in POMs field. 


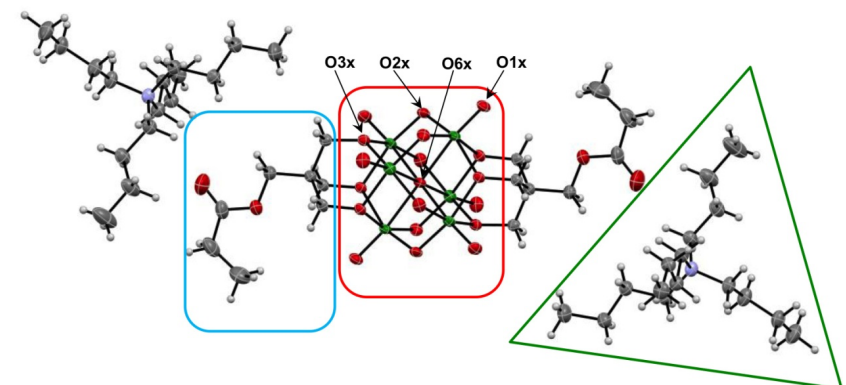

Figure 1. Structure of V6-C3. The red square concerns the V6 core while the blue square concerns the organic moiety. The green triangle indicates the terbutylammonium (TBA) counter cation. See Supplementary information for the detail structure description.

V6-C3 has been synthesized according to the route described by Yin et al.. 12b Structure of the V6-C3 consists of a divalent tris(alkoxo) hexavanadate anion and two tetrabutylammonium (TBA) acting as counter cations (Fig. 1). The valence of oxygen belonging to the V6 core is indicated in Fig. 1. An accurate high resolution $\mathrm{X}$-ray diffraction experiment at $100 \mathrm{~K}$ has been carried out. 16 The V6-C3 electron density was described using the program package MoPro, ${ }^{17}$ which is based on the multipole formalism developed by Hansen and Coppens. 13a Two refinement strategies have been developed: i) refinement strategy 1, where anion and cation are refined separately, implying no charge transfer between anion and cation; ii) refinement strategy 2 , where anion and cation are refined together authorizing charge transfer between anion and cation (For charge density refinement details and AIM charge 18 see Supplementary information).

The three vanadium atoms, which are in an equivalent chemical environment display positive charges. Comparing with the previously studied $\left[\mathrm{V}_{10} \mathrm{O}_{28}\right]^{6-}(\mathrm{V} 10)$ anion ${ }^{19}$, there is only one type of vanadium atom, the VII type, which exists in V6-C3 (see Supplementary information). The average charges of $\mathrm{V}$ atom in functionalized V6 are 1.27 e (strategy 1) and 1.22 e (strategy 2) compared to 1.53 e (strategy 1) in V10. ${ }^{19}$ These values could be compared to the theoretical natural charges of $\mathrm{V}$ atoms (1.53 e and $1.54 \mathrm{e}$ ) obtained for a similar functionalized hexavanadate bearing redox-active ferrocenyl groups at the terminal of organic ligand, $\left(\mathrm{Bu}_{4} \mathrm{~N}\right)_{2}\left[\mathrm{FcC}(\mathrm{O}) \mathrm{NHC}\left(\mathrm{CH}_{2} \mathrm{O}\right)_{3} \mathrm{~V}_{6} \mathrm{O}_{13}\left(\mathrm{OCH}_{2}\right)_{3} \mathrm{CNHC}(\mathrm{O}) \mathrm{Fc}\right]$ (in further text given as Fc-V6) reported by Schulz et al. ${ }^{20}$ For V6$\mathrm{C} 3$, the oxygen atoms have negative AIM charges in the range from -0.63 ot -1.09 e. With regard to the oxygen valence (Fig 1 ), we observe the following decrease in negative charge: $06 x$ $<03 x<02 x<01 x$. This order has already been observed for the V10 anion charge distribution analysis. The experimental results obtained in V6-C3 and V10 19 are in good agreement with the theoretical Mulliken modified charges determined by Kempf et al. ${ }^{21}$ [06x $(-1.27)<03 x(-1.04)<02 x(-0.90)<01 \mathrm{x}$ $(-0.65)]$ for a V10 anion, and also with natural charges calculated by Schulz et al 20 on a functionalized V6 [06x (-1.11) < $03 \mathrm{x}(-0.74)<02 \mathrm{x}(-0.68)<01 \mathrm{x}(-0.54)]$. Additionally, Bridgeman et al. 22 have also reported a similar trend for the oxygen atomic charges in $\left[\mathrm{M}^{\prime} \mathrm{M}_{5} \mathrm{O}_{19}\right]^{3-}$ Lindqvist anion (where $\mathrm{M}=\mathrm{Mo}$ and $\mathrm{W}$ and $\mathrm{M}^{\prime}=\mathrm{V}, \mathrm{Nb}, \mathrm{Ta}$ ), which belongs to the same POM family.

Charge summation of V6 core and organic ligand is given in Supplementary information. At the initial refinement step of strategy 1 , the -2 anionic charges was sheared on each oxygen atom, leading to a value of +0.435 on V6 core and -2.435 e for the organic ligand. The final values at the end of this refinement (strategy 1), where no charge transfer with cation is allowed, indicate a strong polarization of the anion ( -9.75 for the V6 core and +3.78 e for each of the two organic ligands). Additionally, for a better understanding of the charge distribution of the whole molecule in the crystalline state, we have performed a charge density refinement without constraints on charge transfer between anion and cation (strategy 2). Comparing to the results obtained by strategy I, there is an enhancement of the intramolecular charge transfer (ICT), which is indicated by the slightly more negative charge of V6 core and sligthly more positive charge of the organic ligand (see Supplementary Information, Table S4). Consequently, some charges are also transferred from cation to V6 core through several CH...O weak hydrogen bonds. ${ }^{23}$ All these results are in agreement with the fact that V6 core has a strong electroncontaining capacity, which had never been characterized by direct experimental evidence before. An other way to estimate the charge transfer, consists in the determination of integrated source function 24 at a reference point (each bond critical points, BCPs). Summation on organic ligand, V6 core and cations of the each atom percentage contribution is given in Table 1.

According to this method, $87.03 \%$ of the electron density at BCPs in V6 core originates from the V6 core itself, while $8.94 \%$ comes from the organic ligand and $4.03 \%$ from the cations. The percentages on the diagonal of Table 1 originate from the corresponding parts and display the main contributions. While the other contribution $(8.94+4.03=12.07 \%)$ seems small, it nevertheless indicates a delocalization of the density. Such an observation has also been made for a nickel bimetallic complex. 25

Finally, for an overall description of the charge transfer within V6-C3 hybrid in the solid state, it is necessary to determine the electrostatic potential (EP) features. The experimental EP has been calculated using the AIM charges. 26 The EP values at the 3D iso-density surface $\left(0.007\right.$ e $\left.\AA^{-3}\right)$ in the vicinity of the V6 core oxygen atoms are listed in Supplementary information (Table S5). The corresponding EP features, using the colored scale for EP values on the 3D molecular surface of the functionalized V6-C3 are shown in Fig. 2.

The EP values in the vicinity of the three different oxygen atom types (mono, bi, and tri- coordinated) at the molecular surface are $-0.92,-1.18,-1.45$ e $\AA^{-1}$, respectively obtained from the strategy 1 , while, when the charge transfer is pos sible between anion and cation (strategy 2), the values rise to -1.11 , $-1.40,-1.70$ e $\AA^{-1}$, respectively. Despite the fact that the V6 core possesses a less negative charge after the strategy 2 than after strategy 1 ( -9.75 e versus -10.23 e), the V6 core expresses a more negative EP region in strategy 2 . The same trend is observed for the organic ligand (Fig. 2). This result would be compared to the theoretical EP determination on a similar functionalized hexavanadate, Fc-V6. 20 Unfortunately, there is no indication on the color scale on the published result, which only remarks that the V6 core is negative. In order to exhibit graphically the intermolecular charge transfer between the organic and the inorganic ligand of the V6-C3 compound, the EP iso-value surfaces at $\pm 0.01 \mathrm{e}^{-1}$ has been plotted (Fig. 3 ). The red part is localized around the V6 core, which corresponds to the most nucleophilic regions, while the blue part is concentrated over the organic ligand, which represents the most electrophilic regions. This property represents the most important characteristics of the hybrid compound. 
Table 1. Integrated Source Function (strategy 2)

\begin{tabular}{|c|c|c|c|}
\hline & $\begin{array}{c}\text { Atoms } \\
\text { from cores }\end{array}$ & $\begin{array}{c}\text { Atoms from } \\
\text { organic moiety }\end{array}$ & $\begin{array}{c}\text { Atoms from } \\
\text { Cation }\end{array}$ \\
\hline CPs on Core & $87.03 \%$ & $8.94 \%$ & $4.03 \%$ \\
\hline CPs on OL & $8.64 \%$ & $89.90 \%$ & $1.46 \%$ \\
\hline CPs on Cation & $-0.53 \%$ & $0.76 \%$ & $99.77 \%$ \\
\hline
\end{tabular}

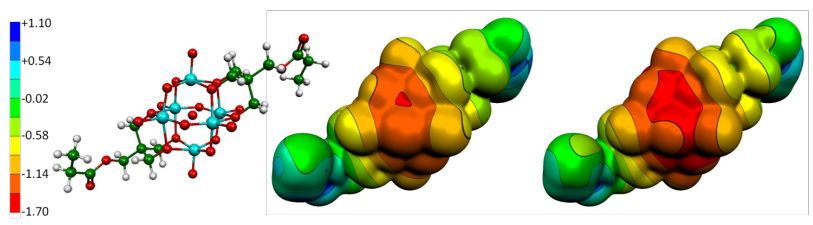

Figure 2. Experimental EP on the molecular surface $(0.007$ $\mathrm{e}^{-3}$ ) a) structure b) strategy $1 \mathrm{c}$ ) strategy 2 .

These observations constitute the first experimental description of a POM-based hybrid at the electronic level. Dolbecq et al. 7 in their review on hybrid organic-inorganic POM compounds have divided them in two classes: i) class I, where the connections between organic and inorganic part are done through electrostatic, hydrogen bonds or van der Waals interactions; ii) class II, where connections are done through covalent or iono-covalent bonds. Fig. 4a represents the static deformation density in the $\mathrm{V}-\mathrm{O} 3 \mathrm{x}-\mathrm{C}$ plane. The Laplacian of electron density in the same plane is given in Fig. $4 \mathrm{~b}$. The green arrow indicates the polarizat ion of the $03 x$ in the direction of the vanadium atom while the purple arrow indicates the polarization in the direction of the carbon atom. This anisotropic behavior is a clear signature of the bonds toward the inorganic V6 core and toward the organic ligand. The AIM analysis gives $(3,-1)$ bond critical points (BCPs) for each $V-03 x$ bonds (average $\rho$ values $=0.54$ e. $\AA^{-3}$ ). The energy density $(H)$ has been determined according to the Abramov's formula 27 and is plotted in Fig. 4c versus the different V-O distances from V6-C3 and V10 anion. Therefore, the functionalized hexavanadate V6-C3 can be declared as an organic-inorganic POM of class II. The 03x form a covalent bond between V6 cluster and organic ligand. Furthermore, the integrated source function at the BCPs of $\mathrm{V}-\mathrm{O} 3 \mathrm{x}$ indicates the contribution of organic and inorganic part. Therefore, for a precise characterization of the functionalized hexavanadate, it is better to use the term moiety instead of ligand. Except for the V-O6x for which the Laplacian is sometimes positive, the other V-O bonds present a negative Laplacian and consequently a certain covalent character. It has to be noticed that the V6-03x bond has a slightly lower average energy density $H$ than the corresponding one in V10. This is the consequence of the link with the organic moiety.

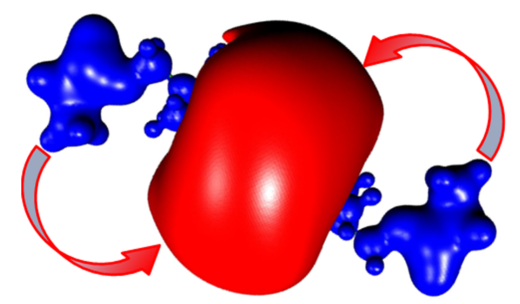

Figure 3. Experimental EP at isovalue sruface $\left( \pm 0.01\right.$ e. $\left.\AA^{-1}\right)$ (strategy 2).

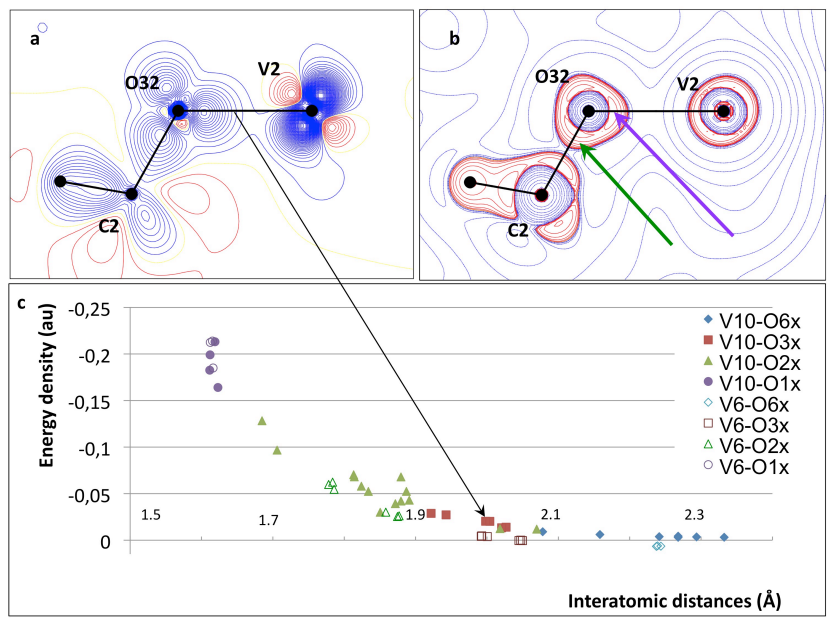

Figure 4. Connection between organic and inorganic part a) Static deformation density (contours $0.05 \mathrm{e}^{-3}$ ) in the V1-031C1 plane b) Laplacian in the same plane. Contribution of the O3x to the V-O3x bond (green arrow) and to the $03 x-C$ bond (purple arrow). c) Relationship between interatomic distances $(\AA)$ and energy density (a.u) for the V-O bond in V6-C3 (unfilled marks) and V10 (plain marks). 19

POMs and POVs have long been considered as the luminescence quenchers. ${ }^{28}$ However, a few examples have shown that these materials exhibit fluorescence. Especially the POV-based materials give the blue luminescent emission in solution.14, The oxide-to-vanadium charge transfer and intervalence charge transfer could be the probable reasons. Recently, functionalized V6 with hydrophobic groups shows unexpected blue luminescence, probably due to the ligand-to-metal charge transfer (LMCT), which might inspire a new mechanism for fluorescence emission. ${ }^{12}$ The V6-C3 compound in the solid state and at the ground state could be described as a donor acceptor according to a $\mathrm{D}^{+}-\mathrm{A}^{2} \quad--\mathrm{D}$ + formula. This indicates that the compound is extremely polarizable in the ground state. One could use the term of intramolecular charge transfer compound to describe the ground state in the solid state of the functionalized V6 species. The functionalized V6 anion exhibits a push-pull behavior. This experimental description of charge density analysis in the ground state is the first step for understanding of the fluorescent properties. HOMO and LUMO are involved in the mechanism of the fluorescent properties observed experimentally by Yin et al.12a in solvent state. There is a small amount of compound for which the experimental X-ray diffraction could be used to access the exited state. ${ }^{29}$ Therefore our work presented in this communication constitutes a reference point for performing accurate DFT theoretical calculations of the two states and therefore explanation on the fluorescent properties of hybrid compounds. Such calculations are actually in progress in our Laboratory.

Electronic supplementary information (ESI) available Experimental methods, crystallographic details, structural description, residual density, Kappa $(\kappa)$ and multipole $\left(\kappa^{\prime}\right)$ coefficient, experimental multipole charge and AIM charge, experimental electrostatic potential. This material is available free of charge via the Internet at www.rsc.org/pccp.

\section{AUTHOR INFORMATION}

\section{Corresponding Author}

anne.spasojevic@centralesupelec.fr 


\section{Notes}

The authors declare no competing financial interest.

\section{ACKNOWLEDGMENT}

The authors thanks Slimane Dahoui, (CRM2, Institut Jean Barriol, UMR7036 CNRS, University de Lorraine, France for his contribution to the X-ray diffraction measurement. XX thanks the China Scholarship Council (CSC) for funding his PhD. SN and NBP thank CentraleSupelec for several invited associate professor positions. NBP would thank the Ministry of Education and Science of the Republic of Serbia for supporting the project (Project OI 172043). PFW and XX thank The Natural Science Foundation of China (NFSC) (no. 21271068) the Natural Science Foundation of Hubei Province (no. 2015CFA131), and the Wuhan Applied Basic Research Program (no. 2014010101010020) for financial support.

\section{REFERENCES}

[1] X. Zhu, Q. Yang and M. Muntwiler, Acc. Chem. Res. 2009, 42, 17791787.

[2] Y. Zhao and W. Liang, Chem. Soc. Rev. 2012, 41, 1075-1087.

[3] H. Miyasaka, Acc. Chem. Res. 2013, 46, 248-257.

[4] Z.R. Grabowski, K. Rotkiewicz and W. Rettig, Chem. Rev. 2003, 103, 3899-4032.

[5] (a) A. V. Akimov, A. J. Neukirch and O. V. Prezhdo, Chem. Rev. 2013, 113, 4496-4565. (b) M. Sadakane and E. Steckhan, Chem. Rev. 1998, 98, 219-237.

[6] (a) R. S.Mulliken, J. Phys. Chem. 1952, 56, 801-822. (b) R. S. Mulliken, Chim. Phys., Chim. Biol. 1964, 61, 20-38. (c) R. S. Mulliken and W. B. Person, Annu. Rev. Phys. Chem. 1962, 13, 107-126. (d) L. E. Orgel and R. S. Mulliken, J. Am. Chem. Soc. 1957, 79, 4839-4846.

[7] A. Dolbecq, E. Dumas, C. R. Mayer and P. Mialane, Chem. Rev. 2010, 110, 6009-6048.

[8] (a) T. Yamase, Chem. Rev. 1998, 98, 307-325. (b) E. Coronado, C. Gimenzsaiz and C. Gomezgarcia, Coord. Chem. Rev. 2005, 249, 17761796.

[9] P L Maguerès, S. M. Hubig, S. V. Lindeman, P. Veya and J. K. Kochi, J. Am. Chem. Soc. 2000, 122, 10073-10082.

[10] (a) R. Tsunashima, T. Matsumoto, N. Hoshino, W. Niiho, M. Kimura, K. Kondo, Y. Suyama, Y. Nishioka, J. Kawamata, S. Noro, T. Nakamura, T. Akutagawa and K. Ishiguro, Dalton Trans. 2012, 41, 1006010064. (b) Q. Li,C. Zhao, A. M. Bond, J. F. Boas, A. G. Wedd, B. Moubaraki and K.S. Murray, J. Mater. Chem. 2011, 21, 5398-5407.

[11] (a)C. Daniel and H. J. Hartl, Am. Chem. Soc. 2005, 127, 1397813987. (b) C. Daniel and H. J. Hartl,. Am. Chem. Soc. 2009, 131, 51015114. (c) C. Aronica, G. Chastanet, E. Zueva, S. A. Borshch, J. M. Clemente-Juan and D. J. Luneau, Am. Chem. Soc. 2008, 130, 2365-2371. (d) J. Spandl, C. Daniel, I. Brudgam and H. Hartl, Angew. Chem. Int. Ed. 2003, 42, 1163-1166.

[12] (a) P. Yin, P. Wu, Z. Xiao, D. Li, E. Bitterlich, J. Zhang, P. Cheng, D. V. Vezenov, T. Liu and Y. Wei, Angew. Chem. Int. Ed. 2011, 50, 25212525. (b) P. Wu, Z. Xiao, J. Zhang, J. Hao, J. Chen, P. Yin and Y. Wei, Chem. Commun. 2011, 47, 5557-5559. (c) D. Li, J. Song, P. Yin, S.
Simotwo, A. J. Bassler, Y, Aung, J. E. Roberts, K. I. Hardcastle, C. L. Hill and T. Liu, J. Am. Chem. Soc. 2011, 133, 14010-14016.

[13] (a) N. K. Hansen and P. Coppens, Acta Crystallogr. 1978, A34, 909-921. (b) T. S. Koritsanszky and P. Coppens, Chem. Rev. 2001, 101 1583-1628. (c) R. F. W. Bader, Chem. Rev. 1991, 91, 893-928. (d) C. Gatti and L. Bertini, Acta Crystallogr. 2004, A60, 438-449. (e) C. Lecomte, M. Souhassou and S. Pillet, J. Mol. Struct. 2003, 647, 53-64.

[14] (a) H. Ma, X. Meng, J. Sha, H. Pang and L. Wu, Solid State Sci. 2011, 13, 850-854. (b) L. Chen, F. Jiang, N. Li, C. Yan, W. Xu and M. Hong, Inorg. Chem. Commun. 2009, 12, 219-222. (c) L. Chen, F. Jiang, Z. Lin, Y. Zhou, C. Yue and M. Hong, J. Am. Chem. Soc. 2005, 127, 8588-8589. (d) T. Yamase and K. Ohtaka, J. Chem. Soc. Dalton Trans. 1994, 18 2599-2608. (e) XL Wang, JJ. Sun, HY. Lin, ZH. Chang, GC. Liu, Inorg. Chem. Com. 2016, 73, 152-156

[15] (a) I. Ortmann, S. Werner, C. Kruger, S. Mohr and K. Schaffner, J. Am. Chem. Soc. 1992, 114, 5048-5054. (b) K. Meindl, J. Henn, N.Kocher, D. Leusser, K. A. Zachariasse, G. M. Sheldrick, T. Koritsanszky and D. Stalke, J. Phys. Chem. A. 2009, 113, 9684-9691. (c) 0. Kotova, K. Lyssenko, A. Rogachev, S. Eliseeva, I. Fedyanin, L. Lepnev, L. Pandey, A. Burlov, A. Garnovskii, A. Vitukhnovsky, M. Van der Auweraer and N. Kuzmina, J. Photochem. Photobiol. A. 2011, 218, 117-129.

[16] For crystallographic details see Supplementary information.

[17] (a) B. Guillot, L. Viry, R. Guillot, C. Lecomte and C. Jelsch, J. Appl. Crystallogr. 2000, 34, 214-223. (b)C. Jelsch, B. Guillot, A. Lagoutte and C. Lecomte, J. Appl. Crystallogr. 2005, 38, 38-54.

[18] R. F. W. Bader, Atoms in Molecules: A Quantum Theory; Claredon Press: Oxford, 1990

[19] N. Bošnjaković-Pavlović, A. Spasojević - de Biré, I. Tomaz, N. Bouhmaida, F. Avecilla, U. Mioc, J.C.Pessoa and N.E. Ghermani, Inorg. Chem. 2009, 48, 9742-9753

[20] J. Schulz, R. Gyepes, I. Císařová and P. Štěpnička, New J. Chem. 2010, 34, 2749-2756.

[21] J. Y. Kempf, M. M. Rohmer, J. M. Poblet, C. Bo and M. Bénard, J. Am. Chem. Soc. 1992, 114, 1136-1146.

[22] A. Bridgeman and G. Cavigliasso, Faraday Discuss. 2003, 124, 239-258.

[23] Due to the huge size of the compound, the AIM charges calculation failed to reach the electroneutrality within $-0.58 / 0.52$ e. Different calculation using different codes have given similar values. $0.58 /$ 0.52 e corresponds to $0.08 \% / 0.07 \%$ of the F000. From a crystallographic point of view only $0,29 / 0.26$ e failed to reach the electroneutrality.

[24] (a) R. F. W. Bader and C. Gatti, Chem. Phys. Lett. 1998, 287, 233

238. (b) C. Gatti and F. Cargnoni, L. Bertini, J. Comput. Chem. 2003, 24, 422-436.

[25] R. Kaminski, B. Herbaczynska, M. Srebro, A. Pietrzykowski, A. Michalak, L. Jerzykiewicz and K. Wozniak, Phys. Chem. Chem. Phys. 2011, 13, 10280-10284.

[26] N. Bouhmaida and N. E. Ghermani, N. E. J. Chem. Phys. 2005, 122, 114101.

[27] Y. A. Abramov, Acta Crystallogr. 1997, A53, 264-272.

[28] T. M. Anderson, W. A Neiwert, M. L. Kirk, P. M. B. Piccoli, A. J. Schultz, T. F. Koetzle, D. G. Musaev, K. Morokuma, R. Cao, C. L. Hill, Science, 2004, 306, 2074- 2077

[29] a) B. Fournier and. Coppens, J. Synchr Rad., 2012, 19, 497-502. 